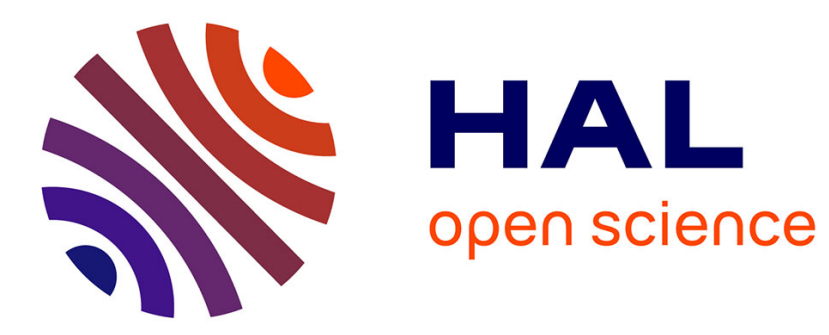

\title{
Études expérimentales sur un prisme magnétique
}

J. Camplan, R. Meunier

\section{To cite this version:}

J. Camplan, R. Meunier. Études expérimentales sur un prisme magnétique. Revue de Physique Appliquée, 1969, 4 (2), pp.214-214. 10.1051/rphysap:0196900402021400 . jpa-00243225

\section{HAL Id: jpa-00243225 https://hal.science/jpa-00243225}

Submitted on 1 Jan 1969

HAL is a multi-disciplinary open access archive for the deposit and dissemination of scientific research documents, whether they are published or not. The documents may come from teaching and research institutions in France or abroad, or from public or private research centers.
L'archive ouverte pluridisciplinaire HAL, est destinée au dépôt et à la diffusion de documents scientifiques de niveau recherche, publiés ou non, émanant des établissements d'enseignement et de recherche français ou étrangers, des laboratoires publics ou privés. 


\title{
ÉTUDES EXPÉRIMENTALES SUR UN PRISME MAGNÉTIQUE
}

\author{
J. GAMPLAN et R. MEUNIER, \\ Centre de Spectrométrie Nucléaire et de Spectrométrie de Masse du C.N.R.S., Orsay.
}

\begin{abstract}
Résumé. - Deux méthodes expérimentales d'étude d'un prisme magnétique sont décrites. La première conduit à la connaissance des indices du champ et à la détermination de shims, la seconde permet de déterminer un profil d'entrée qui réduit la largeur des raies.
\end{abstract}

\begin{abstract}
Two experimental methods concerning a magnetic prism are described. They lead : the first one to $i$ ) the field indexes ; ii) the determination of shims ; the second one to an entrance profile which reduces the image width.
\end{abstract}

Le prisme que nous avons étudié est destiné à un séparateur d'isotopes et doit satisfaire à de nombreuses exigences. Il doit, entre autres choses : i) créer une dispersion assez grande afin de faciliter la construction des collecteurs; nous avons donc utilisé un prisme à champ inhomogène de rayon moyen $r_{0}=85 \mathrm{~cm}$; ii) donner des raies très fines afin d'augmenter la pureté isotopique des produits recueillis. Ce dernier point nous a conduits à :

1) déterminer des shims circulaires qui élargissent la zone utile de l'entrefer. Pour cela, nous avons utilisé une méthode [1] qui consiste essentiellement à déplacer une bobine d'un mouvement uniforme le long d'un rayon. L'enregistrement de la f.e.m. qui apparaît aux bornes de cette bobine permet et de connaitre les indices du champ et de juger de l'effet de shims placés localement autour de la région étudiée. Ainsi l'effet des shims circulaires peut être étudié directement sur l'aimant (donc en particulier à plusieurs inductions), sans qu'il soit nécessaire de les réaliser complètement. Gette méthode simple et peu coûteuse nous a permis de connaitre les indices du champ avec une précision de $\pm 3 \%$ pour le premier et de $\pm 6 \%$ pour le second. Les shims circulaires ainsi déterminés réduisent à $8 \mathrm{~cm}$ vers l'extérieur et à $6 \mathrm{~cm}$ vers l'intérieur la zone inutilisable de l'entrefer qui a $40 \mathrm{~cm}$ de large et $15 \mathrm{~cm}$ de haut.

2) Étudier, au voisinage du point de focalisation, les positions relatives de sept trajectoires uniformément réparties dans un faisceau d'ions défléchis par le prisme [2] (ces sept trajectoires sont matérialisées par les ombres de sept fils, régulièremęnt espacés, placés à l'entrée de l'aimant, parallèlement aux lignes de champ). En plaçant des pièces de fer sur la face d'entrée du prisme, on modifie aisément les positions relatives de ces trajectoires. Ainsi nous avons pu déterminer trois profils de face d'entrée qui donnent, quelle que soit l'induction, des raies de largeur inférieure à $0,3 \mathrm{~mm}$. Ces profils d'entrée donnent au prisme magnétique un pouvoir de résolution voisin de 10000 à mi-hauteur de pic. De plus, les coefficients d'enrichissement obtenus avec ce séparateur sont égaux ou même plus élevés que ceux obtenus avec le séparateur à deux étages précédemment construit [3].

\section{BIBLIOGRAPHIE}

[1] Camplan (J.) et Meunier (R.), Nucl. Instr. Methods, 1964, 31, 192.

[2] Camplan (J.) et Meunier (R.), Nucl. Instr. Methods, 1967, 57, 252.

[3] Bernas (R.), Camplan (J.) et Sarrouy (J. L.), J. Physique Rad., 1960, 21, 191. 